\title{
Risk Factors for Generalization in Patients with Ocular Myasthenia Gravis: A Multicenter Retrospective Cohort Study
}

\author{
Rong-Jing Guo $\cdot$ Ting Gao $\cdot$ Zhe Ruan $\cdot$ Hong-Yu Zhou $\cdot$ Feng Gao $\cdot$ Quan Xu $\cdot$ Li-Ping Yu $\cdot$ Song-Di Wu $\cdot$ \\ Tao Lei $\cdot$ Huan-Huan Li $\cdot$ Chao Sun · Min Zhang $\cdot$ Yan-Wu Gao $\cdot$ Xiao-Dan Lu $\cdot$ Yong-Lan Tang • \\ Bao-Li Tang $\cdot$ Fei-Yan Huo $\cdot$ Ying Zhu $\cdot$ Zhu-Yi Li $\cdot$ Ting Chang (D)
}

Received: August 20, 2021 / Accepted: October 14, 2021 / Published online: November 2, 2021

(c) The Author(s) 2021

\begin{abstract}
Introduction: Many patients with ocular myasthenia gravis (OMG) progress to generalized disease within the first 2 years of the onset of ocular symptoms. Several retrospective studies have identified risk factors associated with generalization, however these studies included patients on immunosuppression therapy or
\end{abstract}

Rong-Jing Guo, Ting Gao, Zhe Ruan, Hong-Yu Zhou and Feng Gao are co-first authors.

Supplementary Information The online version contains supplementary material available at https:// doi.org/10.1007/s40120-021-00292-x.

R.-J. Guo - T. Gao - Z. Ruan · H.-H. Li · C. Sun ·

M. Zhang · Y.-W. Gao · X.-D. Lu · Y.-L. Tang ·

B.-L. Tang · F.-Y. Huo · Y. Zhu · Z.-Y. Li (৫) .

T. Chang $(\varangle)$

Department of Neurology, Tangdu Hospital, The

Fourth Military Medical University, Xi'an 710038,

China

e-mail: lizhuyiafu@163.com

T. Chang

e-mail: changting1981@163.com

\section{H.-Y. Zhou}

Department of Neurology, West China Hospital,

Sichuan University, Chengdu, China

F. Gao

Department of Neuroimmunology, Henan Institute of Medical and Pharmaceutical Sciences, Zhengzhou University, Zhengzhou, China those undergoing thymectomy, which may reduce the generalization risk. In this study we explored the risk factors for generalization in non-immunosuppressed and non-thymectomized patients with OMG.

Methods: Data from patients with OMG treated at seven tertiary hospitals in China were retrospectively reviewed. Clinical characteristics, including sex, age at onset, symptoms at onset, comorbid autoimmune diseases, neostigmine test response, repetitive nerve stimulation (RNS) findings, presence of serum anti-acetylcholine receptor antibody (AChR-Ab), and thymic status based on radiological and pathological studies, were collected. The main outcome measure was disease generalization. The follow-

Q. Xu

Department of Thoracic Surgery, Jiangxi Provincial People's Hospital, Nanchang, China

L.-P. Yu

Department of Neurology, Xianyang First People's Hospital, Xianyang, China

S.-D. Wu

Department of Neurology, Xi'an No.1 Hospital, Xi'an, China

T. Lei

Department of Neurology, Xi'an Fourth Hospital, Xi'an, China 
up period was defined as the date of ocular symptom onset to the date of confirmation of generalization or immunotherapy initiation, or last follow-up (defined as 60 months). The Cox proportional hazards model was used to assess the risk factors for generalization.

Results: Overall, 572 patients (269 women) were eligible for inclusion in the analysis, of whom 144 developed generalization. The mean (standard deviation) onset age was 45.5 (19.8) years, and the median (interquartile range) follow-up period was 14.5 (7.0-47.3) months. Multivariable Cox regression analysis demonstrated that both early-onset (adjusted hazard ratio [aHR] 5.34; 95\% confidence interval [CI] 1.64-17.36; $p=0.005$ ) and late-onset (aHR 7.18; 95\% CI 2.22-23.27; $p=0.001$ ) in adulthood, abnormal RNS findings (aHR 3.01; 95\% CI $1.97-4.61 ; p<0.001)$, seropositivity for AChR$\mathrm{Ab}$ (aHR 2.58; 95\% CI 1.26-5.26; $p=0.01$ ), and thymoma (aHR 1.62; 95\% CI 1.05-2.49; $p=0.03$ ) were independently associated with increased risk of generalization.

Conclusion: The risk of generalization increased significantly in patients with adultonset OMG, abnormal RNS findings, seropositivity for AChR-Ab, and thymoma, suggesting that these risk factors may predict $O M G$ generalization.

Keywords: Ocular myasthenia gravis; Generalized myasthenia gravis; Generalization; Risk factors; Cox proportional hazards model

\section{Key Summary Points}

\section{Why carry out this study?}

Several retrospective studies have identified risk factors associated with generalization in patients with ocular myasthenia gravis (OMG); however, these studies included patients on immunosuppression therapy or those undergoing thymectomy, which may reduce the generalization risk.

Few studies have been conducted to explore risk factors in immunosuppression-naïve patients.

This study is the first to explore risk factors of generalization in immunosuppressionnaïve OMG patients.

By excluding this important confounding factor and involving a large sample of patients, the findings and conclusions of the present study are more rigorous.

\section{What was learned from the study?}

In this multicenter retrospective cohort study, the risk of generalization increased in patients with adult-onset $\mathrm{OMG}$, abnormal repetitive nerve stimulation findings, seropositivity for antiacetylcholine receptor antibody, and thymoma, providing predictors of disease generalization.

The development of a risk prediction model is warranted to aid early screening of patients at high risk of generalization and enable early initiation of riskmodifying therapy.

\section{INTRODUCTION}

Myasthenia gravis (MG) is an acquired autoimmune disease that targets the neuromuscular junction and which is mainly mediated by antiacetylcholine receptor antibody (AChR-Ab). Specific muscle groups are susceptible, 
particularly the extraocular muscles. Disease confined to the ocular muscles is defined as ocular MG (OMG) and results in ptosis and diplopia. Previous studies have shown that 50\% of patients initially present with isolated ocular symptoms $[1,2]$ and that $20-80 \%$ of these patients subsequently progress to generalized MG (GMG), usually within the first 2 years of onset [2]. Once generalized, patients with MG experience limb weakness and dysphagia, which affect their daily life. Respiratory muscle weakness may be life-threatening and require treatment in the intensive care unit. Preventing the progression from OMG to GMG is crucial to disease outcome.

Early immunosuppression therapy with steroids and other non-steroidal immunosuppressants may prevent the progression to GMG $[3,4]$. However, not all patients will ultimately develop generalization, with approximately $20 \%$ of patients not progressing to generalized disease irrespective of immunosuppressive therapy. Identifying the risk factors associated with the progression from OMG to GMG may help stratify patients into low- and high-risk groups and select those patients who are optimal choices for starting risk-modifying treatment.

Several retrospective studies have reported risk factors associated with generalization, however, these studies included patients on immunosuppression therapy or those undergoing thymectomy, which may reduce the generalization risk [2, 5-14]. Thus, a study exploring risk factors in non-immunosuppressed and nonthymectomized patients is required to adequately control for these confounding factors.

The aim of this retrospective study was to review data from patients with OMG treated at seven tertiary hospitals in China and to explore risk factors for generalization in the non-immunosuppressed and non-thymectomized patients.

\section{METHODS}

This multicenter retrospective cohort study reviewed data on consecutive patients with OMG treated at seven tertiary hospitals in
China between 1 January 2015 and 1 May 2019. The data were extracted from the MG database of Tangdu Hospital and other medical centers. The MG database of Tangdu Hospital was created in 2019, and data were collected from electronic medical records of outpatients and inpatients, or from telephone and face-to-face interviews. The condition of patients included in the database is estimated routinely using the Quantitative Myasthenia Gravis score (QMGs), MG-Activities of Daily Living (MG-ADL) score, and MG-Composite (MGC) score, with the patients receiving regular visits, usually at 1 - to 3 -month intervals. Ten neurologists and three nurses participated in data collection, and a total of 103 items were collected, including demographic, clinical, immunological, therapeutic, and thymectomy-related characteristics. The diagnosis of OMG was confirmed by at least two neurologists. Data from other medical centers were obtained through review of the electronic medical records of outpatients and inpatients based on the uniform items.

OMG diagnosis was based on the fluctuating ptosis and/or diplopia at disease onset, with further neurological examination, such as QMGs, suggesting no signs of a generalized disease (i.e., weakness of the limbs or the facial, bulbar, neck, or respiratory muscles), supported by abnormal results from at least one of the following tests: (1) positive response to neostigmine test; (2) abnormal repetitive nerve stimulation (RNS) findings ( $>10 \%$ decrease in the amplitude of the compound muscle action potential upon stimulation of the facial, ulnar, axillary, and accessory nerves); or (3) seropositivity for AChR-Ab. All AChR-Ab tests were performed using an enzyme-linked immunosorbent assay (ELISA) and radioimmunoassay in the Wuhan Kangshengda Medical Laboratory and Henan Institute of Medical and Pharmaceutical Sciences, both in China. Only those patients who had purely ocular symptoms for at least 3 months from onset were included [15]. Chest radiological tests, either computed tomography (CT) or magnetic resonance imaging (MRI), and pathological findings postthymectomy were used to determine a thymoma. 
The exclusion criteria were: (1) having undergone thymectomy within 3 months of ocular symptom onset, or unknown history of thymectomy; (2) use of corticosteroids or other immunosuppressants within 3 months of ocular symptom onset, or unknown immunotherapy status; and (3) missing data on outcomes during the follow-up period.

The follow-up period was defined as the date of ocular symptom onset to the date of generalization confirmation, or immunotherapy initiation, or last follow-up (defined as 60 months). Data prior to immunotherapy initiation were treated as censored data. The outcome measure was disease generalization, defined as the progression of any symptoms or signs involving the facial, bulbar, neck, limb, or respiratory muscles. Based on the development of generalization, the patients were categorized into two groups. Patients whose OMG was maintained and showed disease progression were defined as OMG-M and OMG-G, respectively.

This study was approved by the Ethics Committee of Tangdu Hospital, the Fourth Military Medical University (Approval: No. 202009-14). Research conducted at other medical centers was approved by the respective ethics committee (Electronic Supplementary Material [ESM] Table S1). The written informed consent requirement was waived by the respective ethics committee due to the retrospective nature of the study. This study was conducted and reported in accordance with the Strengthening the Reporting of Observational Studies in Epidemiology (STROBE) reporting guidelines [16]. The study was conducted in accordance with the Helsinki Declaration of 1964 and its later amendments.

\section{Data Collection}

Data on the following baseline variables were collected to identify risk factors for generalization: sex, age at onset, symptoms at onset (ptosis, diplopia), comorbid autoimmune diseases, neostigmine test response, RNS findings, presence of $\mathrm{AChR}-\mathrm{Ab}$, and thymic status as detected by chest radiological test (CT or MRI) and pathological findings post-surgery. Based on the age at onset, patients were categorized as having juvenile-onset ( $<18$ years), early-onset (18-49 years), and late-onset ( $\geq 50$ years) disease. Early- and late-onset OMG are sub-categories of adult-onset OMG [17, 18]. Comorbid autoimmune diseases included systemic lupus erythematosus, multiple sclerosis, rheumatoid arthritis, Hashimoto's thyroiditis, and optic neuromyelitis spectrum disease.

\section{Statistical Analysis}

Age at onset was reported as the mean and standard deviation (SD). Variables reported in the follow-up period were reported as the median and interquartile range (IQR). The other baseline variables were categorical variables and were reported as counts and percentages. Continuous variables were compared between the groups (OMG-M and OMG-G) using the Mann-Whitney $U$ test. For categorical variables, the chi-square or Fisher exact tests were performed to compare the groups. All included baseline variables passed the proportional hazard model hypothesis test. The "ggcoxzph ()" function in the "survminer" package was used to perform a graphical diagnosis, and the test $p$ value of each covariate is given in ESM Fig. S1. Univariable Cox proportional hazards regression models were used to evaluate the associations between candidate risk factors and generalization. Subsequently, variables with $p$ values of $<0.1$ in univariable regression analyses and those recognized as risk factors in the literature were included in the multivariable Cox regression model. Statistical significance was set at $p<0.05$. The Kaplan-Meier curves were used to display the cumulative generalization rates among the different groups. Missing data were accounted for using multiple imputation techniques, and the analysis was repeated in the imputed dataset [19]. All analyses were performed using R statistical software package (version 4.0.0; R Foundation for Statistical Computing, Vienna, Austria). 


\section{RESULTS}

A total of 572 patients with OMG were included in this study. Among these, 144 (25.2\%) developed generalization and 428 (74.8\%) did not during the study period (Fig. 1). The patients' demographic and disease-related characteristics are presented in Table 1. A total of 269 (47.0\%) patients were women. The mean (SD) age at onset was 45.5 (19.8) years, and the median (IQR) follow-up period was 14.5 (7.0-47.3) months. A total of $62(10.8 \%)$ patients had juvenile-onset OMG and 510 (89.2\%) patients had adult-onset OMG. Of the 572 patients, 358 (62.6\%) presented with ptosis only, and 214 (37.4\%) presented with either diplopia or diplopia and ptosis. In addition, 17 (3.0\%) patients had comorbid autoimmune diseases. Regarding testing, 530 patients had performed the AChR-Ab test, 501 patients had performed
RNS test, and 523 patients had performed neostigmine test. OMG diagnosis was based on symptoms and neurological examination, supported by abnormal results from at least one of the following tests: (1) positive AChR-Ab test results (395/530 patients [74.5\%]); (2) abnormal RNS findings (249/501 patients [49.7\%]); and (3) positive response to neostigmine test (505/ 523 patients [96.6\%]). Of the 135 seronegative patients, $132(97.8 \%)$ had performed the RNS test, with 42 of these 132 patients (31.8\%) showing abnormal results; the follow-up period for the remaining 93 patients (of the 35 seronegative patients) was a minimum of 24 months and a maximum of 60 months. Of these 93 patients, three (3.2\%) showed generalization and re-testing of AChR-Ab was positive, 61 (65.6\%) underwent repeated AChR-Ab tests, and 26 showed positive through cell-based assays. In 102 cases of OMG, a thymoma was

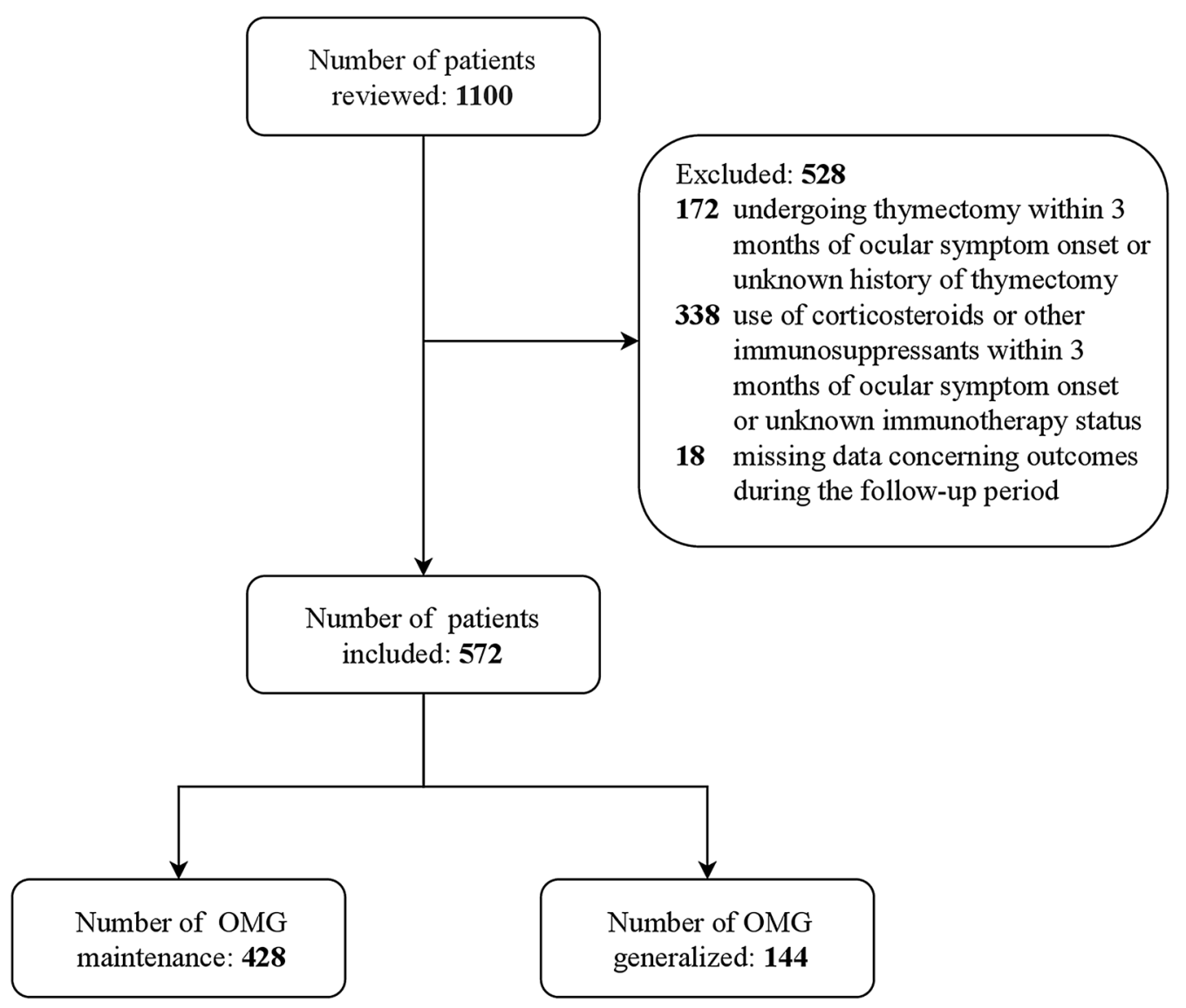

Fig. 1 Flowchart of the study population selection and classification process. $O M G$ Ocular myasthenia gravis 
Table 1 Baseline characteristics of the study population

\begin{tabular}{|c|c|c|c|c|}
\hline Baseline characteristics & $\begin{array}{l}\text { Overall } \\
(n=572)\end{array}$ & $\begin{array}{l}\text { OMG-M group } \\
(n=428)\end{array}$ & $\begin{array}{l}\text { OMG-G group } \\
(n=144)\end{array}$ & $p$ value \\
\hline Sex, $n(\%)$ & & & & 0.27 \\
\hline Male & $303 / 572(53.0)$ & $233 / 428(54.4)$ & $70 / 144(48.6)$ & \\
\hline Female & $269 / 572(47.0)$ & $195 / 428(45.6)$ & $74 / 144(51.4)$ & \\
\hline Onset age, years, mean $\pm S D$ & $(45.5 \pm 19.8)$ & $(42.8 \pm 20.6)$ & $(53.5 \pm 14.8)$ & $<0.001$ \\
\hline Onset age, years, $n(\%)$ & & & & $<0.001^{\mathrm{a}}$ \\
\hline$<18$ & $62 / 572(10.8)$ & $59 / 428(13.8)$ & $3 / 144(2.1)$ & \\
\hline $18-49$ & $236 / 572(41.3)$ & $183 / 428(42.8)$ & $53 / 144(36.8)$ & \\
\hline$\geq 50$ & $274 / 572(47.9)$ & $186 / 428(43.5)$ & $88 / 144(61.1)$ & \\
\hline Symptoms at onset, $n(\%)$ & & & & 0.03 \\
\hline Ptosis & $358 / 572(62.6)$ & $279 / 428(65.2)$ & $79 / 144(54.9)$ & \\
\hline Diplopia/diplopia and ptosis & $214 / 572(37.4)$ & $149 / 428(34.8)$ & $65 / 144(45.1)$ & \\
\hline Comorbid autoimmune diseases, $n(\%)^{\mathrm{b}}$ & & & & 0.78 \\
\hline No & $555 / 572(97.0)$ & $416 / 428(97.2)$ & $139 / 144(96.5)$ & \\
\hline Yes & $17 / 572(3.0)$ & $12 / 428(2.8)$ & $5 / 144(3.5)$ & \\
\hline RNS findings, $n(\%)$ & & & & $<0.001$ \\
\hline Missing & $71 / 572(12.4)$ & $45 / 428(10.5)$ & $26 / 144(18.1)$ & \\
\hline Normal & $252 / 501(50.3)$ & $222 / 383(58.0)$ & $30 / 118(25.4)$ & \\
\hline Abnormal & $249 / 501(49.7)$ & $161 / 383(42.0)$ & $88 / 118(74.6)$ & \\
\hline AChR-Ab, $n(\%)$ & & & & $<0.001^{\mathrm{a}}$ \\
\hline Missing & $42 / 572(7.3)$ & $29 / 428(6.8)$ & $13 / 144(9.0)$ & \\
\hline Seronegative & $135 / 530(25.5)$ & $126 / 399(31.6)$ & $9 / 131(6.9)$ & \\
\hline Seropositive & $395 / 530(74.5)$ & $273 / 399(68.4)$ & $122 / 131(93.1)$ & \\
\hline Neostigmine test, $n$ (\%) & & & & $0.27^{\mathrm{a}}$ \\
\hline Missing & $49 / 572(8.6)$ & $33 / 428(7.7)$ & $16 / 144(11.1)$ & \\
\hline Negative & $18 / 523(3.4)$ & $16 / 395(4.1)$ & $2 / 128(4.7)$ & \\
\hline Positive & $505 / 523(96.6)$ & $379 / 395(95.9)$ & $126 / 128(98.4)$ & \\
\hline Thymic status, $n(\%)$ & & & & 0.002 \\
\hline Missing & $1 / 572(0.2)$ & $1 / 428(0.2)$ & 0 & \\
\hline Non-thymoma & $478 / 571(83.7)$ & $370 / 427(86.7)$ & $108(75.0)$ & \\
\hline Thymoma & $93 / 571(16.3)$ & $57 / 427(13.3)$ & $36(25.0)$ & \\
\hline
\end{tabular}


Table 1 continued

\begin{tabular}{lllll}
\hline Baseline characteristics & $\begin{array}{l}\text { Overall } \\
(\boldsymbol{n}=\mathbf{5 7 2})\end{array}$ & $\begin{array}{l}\text { OMG-M group } \\
(\boldsymbol{n}=\mathbf{4 2 8})\end{array}$ & $\begin{array}{l}\text { OMG-G group } \\
(\boldsymbol{n}=\mathbf{1 4 4})\end{array}$ & $\boldsymbol{p}$ value \\
\hline $\begin{array}{l}\text { Follow-up period duration, months, } \\
\text { median (IQR) }\end{array}$ & $14.5(7.0,47.3)$ & $24.0(8.0,57.0)$ & $12.0(6.0,24.0)$ & $<0.001^{\mathrm{c}}$ \\
\hline
\end{tabular}

$A C h R-A b$ Acetylcholine receptor antibody, IQR interquartile range, $O M G$ ocular myasthenia gravis, $O M G$ - $G$ OMG generalized, $O M G-M$ OMG maintenance, $R N S$ repetitive nerve stimulation, $S D$ standard deviation

${ }^{\mathrm{a}}$ Fisher exact test

${ }^{\mathrm{b}}$ Comorbid autoimmune diseases included systemic lupus erythematosus, multiple sclerosis, rheumatoid arthritis, Hashimoto's thyroiditis, and optic neuromyelitis spectrum disease

'Mann-Whitney $U$ test

suspected based on radiological findings; 97 patients underwent thymectomy and five did not do. Of these 97 patients, 88 (90.7\%) were confirmed to have had a thymoma based on pathological findings post-surgery. A total of 93 patients were ultimately categorized into the thymoma group (Table 1).

Data were not complete for 71 patients in our study cohort. The missing variables included the results of the RNS, AChR-Ab, neostigmine test, and thymic status. The number of missing variables are presented in Table 1 , and the proportion of missing variables was visualized by the "aggr()" function in the "VIM" package R (ESM Fig. S2). The baseline characteristics of imputed datasets was shown in ESM Table S2.

Compared with the OMG-M group, the OMG-G group contained more patients with adult-onset OMG, diplopia as initial symptom, abnormal RNS findings, seropositivity for AChR-Ab, and thymoma $(p<0.05)$. However, there were no significant differences between the groups in terms of sex or comorbid autoimmune disease distribution (Table 1).

The univariable Cox proportional hazards regression model showed that adult-onset OMG, diplopia as initial symptom, abnormal RNS findings, seropositivity for AChR-Ab, and thymoma were significantly associated with the increased risk of generalization (Table 2). Further multivariable Cox regression analysis demonstrated that both early-onset (adjusted hazard ratio [aHR] 5.34; 95\% confidence interval $[\mathrm{CI}] 1.64-17.36 ; p=0.005)$ and late-onset
(aHR 7.18; 95\% CI 2.22-23.27; $p=0.001$ ) in adulthood significantly increased the risk of generalization. Patients with abnormal RNS findings were more likely than their counterparts to progress to generalized disease (aHR 3.01 ; 95\% CI 1.97-4.61; $p<0.001$ ). Seropositivity for AChR-Ab and thymoma were also correlated with an increased risk of generalization (aHR 2.58; 95\% CI 1.26-5.26; $p=0.01$ and a HR $1.62 ; 95 \%$ CI $1.05-2.49 ; p=0.03$, respectively) (Table 3). The results of univariable and multivariable Cox regression analysis in the imputed datasets were consistent with the results without multiple imputations (Tables 2, 3).

The Kaplan-Meier curves demonstrated the cumulative generalization rates of OMG in different groups. Higher cumulative generalization rates were observed in patients with adult-onset, abnormal RNS findings, seropositivity for AChR-Ab and thymoma ( $p<0.001$; Fig. 2A-D).

\section{DISCUSSION}

In this multicenter retrospective cohort study, the risk of generalization increased in OMG patients with adult-onset OMG, abnormal RNS findings, seropositivity for AChR-Ab, and thymoma. This study is first to explore risk factors for generalization in a large sample of immunosuppression-naive OMG patients.

Our study found that patients with adultonset OMG were more likely to develop generalization than those with the juvenile-onset 
Table 2 Risk factors for OMG generalization in univariable Cox proportional hazards regression model

\begin{tabular}{|c|c|c|c|c|}
\hline \multirow[t]{2}{*}{ Risk factors } & \multicolumn{2}{|c|}{ Before multiply-imputed } & \multicolumn{2}{|c|}{ After multiply-imputed } \\
\hline & HR (95\% CI) & $p$ value & HR (95\% CI) & $p$ value \\
\hline \multicolumn{5}{|l|}{ Sex } \\
\hline Male & 1 & & 1 & \\
\hline Female & $1.14(0.82-1.58)$ & 0.44 & $1.14(0.82-1.58)$ & 0.44 \\
\hline \multicolumn{5}{|c|}{ Age at onset, years } \\
\hline$<18$ & 1 & & 1 & \\
\hline $18-49$ & $7.23(2.25-23.18)$ & $<0.001$ & $7.23(2.25-23.18)$ & $<0.001$ \\
\hline$\geq 50$ & $10.89(3.44-34.51)$ & $<0.001$ & $10.89(3.44-34.51)$ & $<0.001$ \\
\hline
\end{tabular}

Symptoms at onset

Ptosis

Diplopia/diplopia and ptosis

Comorbid autoimmune diseases ${ }^{\mathrm{a}}$

No

Yes

RNS findings

\section{Normal}

Abnormal

AChR-Ab

Seronegative

Seropositive

Thymic status

Non-thymoma

Thymoma
1

$1.48(1.07-2.06)$

0.02

1

$1.48(1.07-2.06)$

0.02

1

$0.89(0.37-2.18)$

1

$3.32(2.19-5.03)$

1

5.09 (2.59-10.02)

0.81

1

$0.89(0.37-2.18)$

0.81

$3.51(2.37-5.19)$

$<0.001$

CI Confidence interval, $H R$ hazard ratio

${ }^{a}$ Comorbid autoimmune diseases included systemic lupus erythematosus, multiple sclerosis, rheumatoid arthritis, Hashimoto's thyroiditis, and optic neuromyelitis spectrum disease

form of this disease; moreover, those with lateonset OMG had a higher risk of generalization. This finding is consistent with results from previous studies $[8,13,14,20,21]$. Wang et al. reported the group with secondary generalization in their study comprised more late-onset patients [8]. Feng et al. also found converted OMG patients had an older onset age (threshold: 43 years) [13]. The reported incidence and prevalence of late-onset MG (age of onset $\geq$ 50 years) have steadily increased over the last decades [22]. Recent MG epidemiology data from China also showed the highest incidence of chronic, generalized MG in the age group of 70-74 years, and this trend was also observed in purely ocular MG [23]. The weak correlation between juvenile-onset OMG and generalization risk is consistent with findings from 
Table 3 Risk factors for OMG generalization in multivariable Cox proportional hazards regression model

\begin{tabular}{|c|c|c|c|c|}
\hline \multirow[t]{2}{*}{ Risk factors } & \multicolumn{2}{|c|}{ Before multiply-imputed data } & \multicolumn{2}{|c|}{ After multiply-imputed data } \\
\hline & Adjusted HR (95\% CI) & $p$ value & Adjusted HR (95\% CI) & $p$ value \\
\hline \multicolumn{5}{|l|}{ Onset age, years } \\
\hline$<18$ & 1 & & 1 & \\
\hline $18-49$ & $5.34(1.64-17.36)$ & 0.005 & $6.24(1.93-20.16)$ & 0.002 \\
\hline$\geq 50$ & $7.18(2.22-23.27)$ & 0.001 & $8.57(2.68-27.48)$ & $<0.001$ \\
\hline \multicolumn{5}{|l|}{ Onset symptoms } \\
\hline Ptosis & 1 & & 1 & \\
\hline Diplopia/diplopia and ptosis & $1.16(0.81-1.68)$ & 0.42 & $1.10(0.79-1.54)$ & 0.57 \\
\hline \multicolumn{5}{|l|}{ RNS findings } \\
\hline Normal & 1 & & 1 & \\
\hline Abnormal & $3.01(1.97-4.61)$ & $<0.001$ & $3.25(2.18-4.86)$ & $<0.001$ \\
\hline \multicolumn{5}{|l|}{$\mathrm{AChR}-\mathrm{Ab}$} \\
\hline Seronegative & 1 & & 1 & \\
\hline Seropositive & $2.58(1.26-5.26)$ & 0.01 & $2.75(1.47-5.17)$ & 0.002 \\
\hline \multicolumn{5}{|l|}{ Thymic status } \\
\hline Non-thymoma & 1 & & 1 & \\
\hline Thymoma & $1.62(1.05-2.49)$ & 0.03 & $1.51(1.03-2.21)$ & 0.04 \\
\hline
\end{tabular}

epidemiological studies in Asian countries. In China, Japan, and likely in other East Asian countries, juvenile-onset OMG has been reported as more common than other OMG types, accounting for approximately $50 \%$ of all cases; the corresponding rate in Western countries has been estimated at $10-15 \%[24,25]$. In addition, juvenile-onset OMG in China tends to be nonsevere and to rarely progress to GMG [25-27].

Seropositivity for AChR-Ab as a predictor of generalization is well-documented $[5,7,9-11,13,20,28-30]$. Galassi et al. surveyed a cohort of 175 patients with OMG and compared the outcome of those patients with antibodies to AChR or to MuSK (muscle-specific tyrosine kinase) with those seronegative for both of these antibodies. The results showed gender, anti-AChR positivity, and MuSK positivity were independent predictors of progression [30]. Moreover, Peeler et al. reported an association between high AChR-Ab levels and progression from $\mathrm{OMG}$ to generalized disease [7]. The results of our study are similar, showing that patients with seropositive AChR-Ab had a higher cumulative generalization rate. Unfortunately, we were unable to further analyze the association of AChR-Ab titer with generalization due to limitations in data collection. The number of AChR-Ab-positive cases has been reported to be lower in ocular (40-70\%) than in generalized (85-90\%) patients with OMG [4]. Additionally, the AChR-Ab positivity rate has also been reported to increase with onset age in purely ocular MG, reaching 100\% in OMG patients with disease onset after the age of 70 years [31]. Similar results were found in our study, with patients aged $>70$ years having a AChR-Ab positivity rate of $88.9 \% \quad(48 / 54$ patients). It would be informative to perform AChR Ab-stratified analysis in patients with 

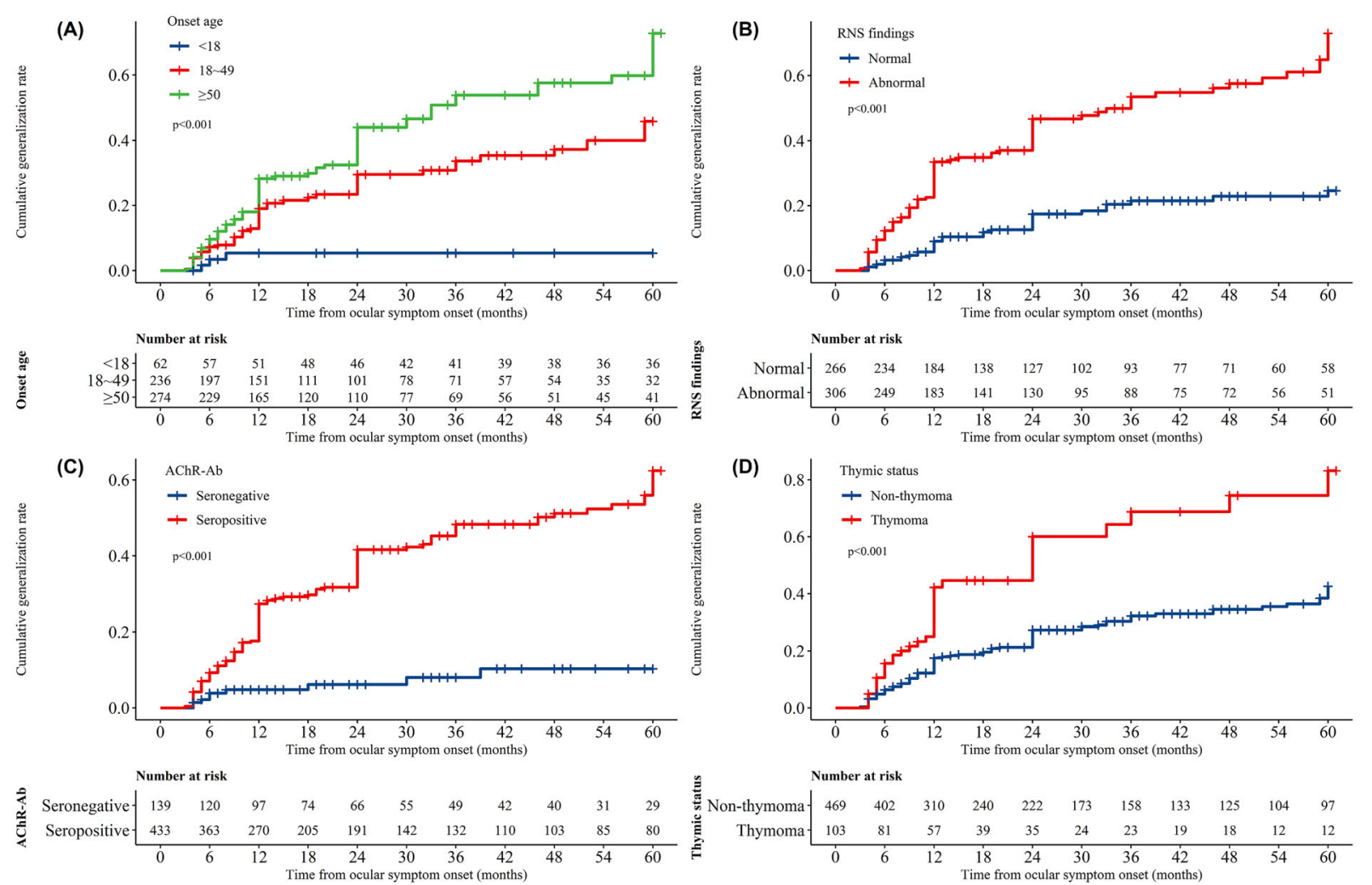

Fig. 2 Kaplan-Meier curves of the cumulative generalization rate from ocular symptom onset in different patient groups. a Patients with juvenile-onset $(<18$ years $)$ and adult-onset OMG (early-onset and late-onset OMG; $\geq 18$ years). b Patients with normal and abnormal RNS findings. c Patients with testing seronegative and

onset at a younger age to determine the predictive value of AChR seropositivity in generalization.

Abnormal RNS findings were found to be an independent risk factor for generalization in our study, which was similar to the results reported by Teo et al. [10]. Approximately $50 \%$ of patients with OMG have positive RNS findings [32]. In our study, $53.5 \%$ of patients presented with abnormal RNS findings, with the OMG-G group containing relatively more patients with positive RNS findings (75\%). The association between nerves in RNS and generalization requires further investigation. To date, two studies have investigated the possible predictive value of different nerves in RNS [5, 14]. Ding et al. found that positive results on the facial RNS test to be a risk factor for generalization but

seropositive for AChR-Ab. d Patients with and without thymoma. Early-onset (18-49 years) and late-onset ( $\geq 50$ years), $A C h R-A b$ Acetylcholine receptor antibody, $R N S$ repetitive nerve stimulation

failed to draw a similar conclusion on the axillary RNS test [14]. Similarly, Hong et al. reported a possibly latent impact of abnormal RNS but had not a predictive value [5]. Single-fiber electromyography (SFEMG), a sensitive test for the diagnosis of OMG, was not performed in our patients due to the inavailability of the test at our center [32]. A previous retrospective study has confirmed the high rate of subclinical SFEMG abnormalities in OMG patients (affecting $68-82 \%$ of cases); however, it failed to show its predictive value [33]. Future studies should study the diagnostic relevance of electrodiagnostic evidence in assessing subclinical generalized disease.

Similar to the results of a study from Korea, thymoma was also found to be a risk factor for generalization [5]. As an immune organ, the 
thymus plays an important role in the pathogenesis of MG. Patients with thymoma tend to have more severe symptoms and even require life-long immunotherapy [34, 35]. Some studies have demonstrated that thymectomy has a possible risk-modifying effect in OMG, further supporting the importance of thymoma as a risk factor $[36,37]$. Given thymoma is modifiable, early thymic surgery may be a therapeutic strategy to decrease the risk of progression to GMG.

Notably, there was a significantly high proportion of purely ocular MG among patients with thymoma-MG in our cohort than has been reported in the literature. Two reasons might contribute to this. First, we considered a minimum of 3 months with purely ocular symptoms as the definition of OMG, so the exclusion of early-generalized patients (within 3 months) would lead to higher ratio of patients with thymoma-OMG. Second, immunotherapy initiation and thymectomy were considered to be signs for stopping the follow-up. Although this study design eliminated the confounding factors derived from surgery and immunotherapy, it also resulted in data that were too highly censored, thereby overestimating the ratio of patients with thymoma-OMG. A total of 216 patients in the OMG-M group were followed up for $>2$ years. Among these patients, the proportion of thymoma-OMG was $10.6 \%(23 / 216$ patients), consistent with the reported frequency of purely ocular $\mathrm{MG}$ in thymoma-MG patients.

Our study has several strengths. First, most previous studies included immunosuppressed patients, potentially affecting the reported outcomes [1, 2, 5-14, 20, 28], as immunosuppression may reduce the risk of generalization $[3,4]$. Excluding this patient group may increase study validity. Second, this study involved a large sample size, which has enabled comprehensive and powerful statistical tests to be performed, yielding rigorous findings and conclusions. For example, a multivariable Cox proportional hazards model was used to handle the censored data. In addition, the up to 60-month follow-up enabled us to capture more outcomes of interest (approx. 25\% of the included cases developed generalization) and obtain robust results.
Some limitations to our study should be considered when interpreting the findings. First, as this was a retrospective study, the present findings may be subject to selection bias and confounding bias. To minimize selection bias, we conducted a multicenter study with a large sample size and used multivariable Cox regression analysis to adjust for confounders. Second, approximately one quarter of patients had negative AChR-Ab findings, and 93 patients showed normal RNS findings. The confirmation of ocular MG diagnosis in these 93 patients relied on the results of the neostigmine test, typical clinical features and patient's response to immunotherapy. Although the results of the neostigmine test are considered to be robust, the assessment may be subjective and prone to false-positive findings. Thus, a misdiagnosis risk might increase in these seronegative patients with normal RNS findings. To minimize misdiagnosis, these 93 patients underwent long-term follow-up (maximum of 60 months and minimum of 24 months) and were re-evaluated every 3 months after the initial assessment. Ultimately, generalization occurred in three patients, and 26 patients re-tested positive for AChR-Ab; no other diagnosis was found in the remaining patients. Third, some data were missing, including the results of the RNS test, $A C h R-A b$, results of the neostigmine test, and thymic status. To reduce the impact of missing data on outcomes, we used chain imputation in the main analysis and performed separate analyses on datasets before and after imputation. Nevertheless, the impact of unmeasured confounders remains exist. Last, patients receiving immunosuppression or thymectomy were excluded from the follow-up, potentially increasing the proportion of censored data and reducing statistical power. Future prospective studies with complete datasets are required to address these limitations.

\section{CONCLUSION}

In summary, the present study has demonstrated that the risk of generalization increased in OM with adult-onset, abnormal RNS findings, seropositivity for AChR-Ab, and thymoma, 
providing predictors of disease generalization. The results strongly suggest that the development of a risk prediction model is warranted to aid early screening of patients at high risk of generalization and to initiate risk-modifying treatment.

\section{ACKNOWLEDGEMENTS}

We thank the patients, their families, and all the investigators involved in this study. We are grateful to co-investigators in the following neuroimmunology teams for their support and help with the study: West China Hospital, Sichuan University, Henan Institute of Medical and Pharmaceutical Sciences, Zhengzhou University, Jiangxi Provincial People's Hospital, Xianyang First People's Hospital, Xi'an No.1 Hospital, and Xi'an Fourth People's Hospital. We also thank Dr. Lin Zeng for the constructive guidance on statistical analysis of this work.

Funding. This work was supported by the National Natural Science Foundation of China (Grant No. 81671233), the discipline innovation and development plan of Tangdu Hospitalmajor program of clinical research (Grant No. 2021LCYJ002). The Rapid Service Fee was funded by the authors.

Authorship. Rong-Jing Guo, Ting Gao, Zhe Ruan, Hong-Yu Zhou, Feng Gao, Quan Xu, LiPing Yu, Song-Di Wu, Tao Lei, Huan-Huan Li, Chao Sun, Min Zhang, Yan-Wu Gao, Xiao-Dan Lu, Yong-Lan Tang, Bao-li Tang, Fei-Yan Huo, Ying Zhu, Zhu-Yi Li, Ting Chang meet the International Committee of Medical Journal Editors (ICMJE) criteria for authorship for this article, take responsibility for the integrity of the work as a whole, and have given their approval for this version to be published.

Authors' Contributions. Conceptualization: Ting Chang, Zhu-Yi Li. Methodology: Ting Chang, Zhu-Yi Li, Rong-Jing Guo, Ting Gao, Zhe Ruan. Formal analysis and investigation: Rong-Jing Guo, Ting Gao, Zhe Ruan, Hong-Yu Zhou, Feng Gao, Quan Xu, Li-Ping Yu, Song-Di
Wu, Tao Lei, Huan-Huan Li, Chao Sun, Min Zhang, Yan-Wu Gao, Xiao-Dan Lu, Yong-Dan Tang, Bao-Li Tang, Fei-Yan Huo, Ying Zhu. Writing (original draft preparation): Rong-Jing Guo, Ting Gao; Writing (review and editing): Ting Chang, Rong-Jing Guo, Ting Gao, Zhe Ruan. Funding acquisition: Ting Chang. Resources: Ting Chang, Zhu-Yi Li, Hong-Yu Zhou, Feng Gao, Quan Xu, Li-Ping Yu, Song-Di $\mathrm{Wu}$, Tao Lei. Supervision: Ting Chang.

Disclosures. Rong-Jing Guo, Ting Gao, Zhe Ruan, Hong-Yu Zhou, Feng Gao, Quan Xu, LiPing Yu, Song-Di Wu, Tao Lei, Huan-Huan Li, Chao Sun, Min Zhang, Yan-Wu Gao, Xiao-Dan Lu, Yong-Lan Tang, Bao-li Tang, Fei-Yan Huo, Ying Zhu, Zhu-Yi Li, Ting Chang declare no potential conflicts of interests.

Compliance with Ethics Guidelines. Patients' records from all medical centers were anonymized and de-identified prior to analysis. This study was approved by the Ethics Committee of Tangdu Hospital, the Fourth Military Medical University (No. 202,009-14). Research conducted at other medical centers was approved by the suitable ethics committee (ESM Table S1). The written informed consent requirement was waived by the respective ethics committee due to the retrospective nature of the study. This study was reported in accordance with the Strengthening the Reporting of Observational Studies in Epidemiology (STROBE) reporting guidelines [16]. The study was conducted in accordance with the Helsinki Declaration of 1964 and its later amendments.

Data Availability. The datasets generated and analyzed during the current study are available from the corresponding author upon reasonable request.

Open Access. This article is licensed under a Creative Commons Attribution-NonCommercial 4.0 International License, which permits any non-commercial use, sharing, adaptation, distribution and reproduction in any medium or format, as long as you give appropriate credit to the original author(s) and the source, provide a link to the Creative Commons licence, and 
indicate if changes were made. The images or other third party material in this article are included in the article's Creative Commons licence, unless indicated otherwise in a credit line to the material. If material is not included in the article's Creative Commons licence and your intended use is not permitted by statutory regulation or exceeds the permitted use, you will need to obtain permission directly from the copyright holder. To view a copy of this licence, visit http://creativecommons.org/licenses/by$\mathrm{nc} / 4.0 /$.

\section{REFERENCES}

1. Li F, Hotter B, Swierzy M, Ismail $M$, Meisel A, Rückert JC. Generalization after ocular onset in myasthenia gravis: a case series in Germany. J Neurol. 2018;265:2773-82.

2. Allen JA, Scala S, Jones HR. Ocular myasthenia gravis in a senior population: diagnosis, therapy, and prognosis. Muscle Nerve. 2010;41:379-84.

3. Li M, Ge F, Guo R, Ruan Z. Do early prednisolone and other immunosuppressant therapies prevent generalization in ocular myasthenia gravis in Western populations: a systematic review and metaanalysis. Ther Adv Neurol Disord. 2019;12:1-8.

4. Kerty E, Elsais A, Argov Z, Evoli A, Gilhus NE. EFNS/ ENS guidelines for the treatment of ocular myasthenia. Eur J Neurol. 2014;21:687-93.

5. Hong YH, Kwon SB, Kim BJ, et al. Prognosis of ocular myasthenia in Korea: a retrospective multicenter analysis of 202 patients. J Neurol Sci. 2008;273:10-4.

6. Nagia L, Lemos J, Abusamra K, Cornblath WT, Eggenberger ER. Prognosis of ocular myasthenia gravis: retrospective multicenter analysis. Ophthalmology. 2015;122:1517-21.

7. Peeler CE, De Lott LB, Nagia L, Lemos J, Eggenberger ER, Cornblath WT. Clinical utility of acetylcholine receptor antibody testing in ocular myasthenia gravis. JAMA Neurol. 2015;72:1170-4.

8. Wang L, Zhang Y, He M. Clinical predictors for the prognosis of myasthenia gravis. BMC Neurol. 2017;17:77.

9. Aguirre F, Villa AM. Prognosis of ocular myasthenia gravis in an Argentinian population. Eur Neurol. 2018;79:113-7.
10. Teo KY, Tow SL, Haaland B, et al. Low conversion rate of ocular to generalized myasthenia gravis in Singapore. Muscle Nerve. 2018;57:756-60.

11. Hendricks TM, Bhatti MT, Hodge DO, Chen JJ. Incidence, epidemiology, and transformation of ocular myasthenia gravis: a population-based study. Am J Ophthalmol. 2019;205:99-105.

12. Apinyawasisuk S, Chongpison Y, Thitisaksakul C, Jariyakosol S. Factors affecting generalization of ocular myasthenia gravis in patients with positive acetylcholine receptor antibody. Am J Ophthalmol. 2020;209:10-7.

13. Feng $X$, Huan $X$, Yan $C$, et al. Adult ocular myasthenia gravis conversion: a single-center retrospective analysis in China. Eur Neurol. 2020;83:182-8.

14. Ding J, Zhao S, Ren K, et al. Prediction of generalization of ocular myasthenia gravis under immunosuppressive therapy in Northwest China. BMC Neurol. 2020;20:238.

15. Wong SH, Huda S, Vincent A, Plant GT. Ocular myasthenia gravis: controversies and updates. Curr Neurol Neurosci Rep. 2014;14:421.

16. Vandenbroucke JP, von Elm E, Altman DG, et al. Strengthening the Reporting of Observational Studies in Epidemiology (STROBE): explanation and elaboration. Int J Surg. 2014;12:1500-24.

17. Popperud TH, Boldingh MI, Rasmussen M, Kerty E. Juvenile myasthenia gravis in Norway: clinical characteristics, treatment, and long-term outcome in a nationwide population-based cohort. Eur J Paediatr Neurol. 2017;21:707-14.

18. Gilhus NE. Myasthenia gravis. N Engl J Med. 2016;375:2570-81.

19. Li P, Stuart EA, Allison DB. Multiple imputation: a flexible tool for handling missing data. JAMA. 2015;314:1966-7.

20. Mazzoli M, Ariatti A, Valzania F, et al. Factors affecting outcome in ocular myasthenia gravis. Int J Neurosci. 2018;128:15-24.

21. Bever CT Jr, Aquino AV, Penn AS, Lovelace RE, Rowland LP. Prognosis of ocular myasthenia. Ann Neurol. 1983;14:516-9.

22. Maddison P, Ambrose PA, Sadalage G, Vincent A. A prospective study of the incidence of myasthenia gravis in the East Midlands of England. Neuroepidemiology. 2019;53:93-9.

23. Chen J, Tian DC, Zhang C, et al. Incidence, mortality, and economic burden of myasthenia gravis 
in China: a nationwide population-based study. Lancet Reg Health West Pac. 2020;5:100063.

24. Evoli A. Acquired myasthenia gravis in childhood. Curr Opin Neurol. 2010;23:536-40.

25. Huang X, Liu WB, Men LN, et al. Clinical features of myasthenia gravis in southern China: a retrospective review of 2,154 cases over 22 years. Neurol Sci. 2013;34:911-7.

26. Gilhus NE, Tzartos S, Evoli A, Palace J, Burns TM, Verschuuren J. Myasthenia gravis. Nat Rev Dis Primers. 2019;5:30.

27. Huang X, Li Y, Feng H, Chen P, Liu W. Clinical characteristics of juvenile myasthenia gravis in Southern China. Front Neurol. 2018;9:77.

28. Kupersmith MJ, Latkany R, Homel P. Development of generalized disease at 2 years in patients with ocular myasthenia gravis. Arch Neurol. 2003;60: 243-8.

29. Sommer N, Sigg B, Melms A, et al. Ocular myasthenia gravis: response to long-term immunosuppressive treatment. J Neurol Neurosurg Psychiatry. 1997;62:156-62.

30. Galassi G, Mazzoli M, Ariatti A, Kaleci S, Valzania F, Nichelli PF. Antibody profile may predict outcome in ocular myasthenia gravis. Acta Neurol Belg. 2018;118:435-43.

31. Monte G, Spagni G, Damato V, Iorio R, Marino M, Evoli A. Acetylcholine receptor antibody positivity rate in ocular myasthenia gravis: a matter of age? J Neurol. 2021;268:1803-7.

32. Al-Haidar M, Benatar M, Kaminski HJ. Ocular myasthenia. Neurol Clin. 2018;36:241-51.

33. O'Hare M, Doughty C. Update on ocular myasthenia gravis. Semin Neurol. 2019;39:749-60.

34. Gilhus NE, Skeie GO, Romi F, Lazaridis K, Zisimopoulou P, Tzartos S. Myasthenia gravis-autoantibody characteristics and their implications for therapy. Nat Rev Neurol. 2016;12:259-68.

35. Romi F, Hong Y, Gilhus NE. Pathophysiology and immunological profile of myasthenia gravis and its subgroups. Curr Opin Immunol. 2017;49:9-13.

36. Geng Y, Dong J, Zhou Q. Rapid improvement of muscle weakness post-thymectomy indicates good long-term neurological outcome in patients with ocular myasthenia gravis. Eur J Neurol. 2019;26: 1421-3.

37. Takanami I, Abiko T, Koizumi S. Therapeutic outcomes in thymectomied patients with myasthenia gravis. Ann Thorac Cardiovasc Surg. 2009;15:373-7. 